\title{
Analytic distribution functions for an ion plasma crossing an MHD shock
}

\author{
M. Siewert and H.-J. Fahr \\ Argelander Institut für Astronomie der Universität Bonn, Abteilung f. Astrophysik und Extraterrestrische Forschung, \\ Auf dem Hügel 71, 53121 Bonn, Germany \\ e-mail: msiewert@astro.uni-bonn.de
}

Received 11 August 2006 / Accepted 24 October 2006

\begin{abstract}
Context. Very recently, we derived the Boltzmann equation for a collisionless plasma crossing an MHD shock, such as that found in astrophysical situations.

Aims. We aim to solve the Boltzmann equation analytically, to obtain exact downstream velocity distribution functions for a given upstream distribution function and an MHD compression ratio.

Methods. We apply the general Boltzmann equation to the case of a purely perpendicular shock and solve it using a separation Ansatz. Results. We obtain a simple, analytical relation between the upstream and downstream velocity distribution functions, which is valid for all physical plasmas. Having obtained the desired relation, we also derive an analytical relation for MHD velocity moments of arbitrary order.
\end{abstract}

Key words. plasma - shock waves - magnetohydrodynamics (MHD) - Sun: solar wind

\section{Introduction}

Magnetohydrodynamics (MHD) is the most successful theory so far capable of describing astrophysical shocks, such as the solar wind termination shock, planetary bowshocks, or supernova shock waves. The shocks are usually described in the form of jump conditions, which are based on the conservation of certain flows of physical quantities, such as the mass flow, the momentum, and the energy flows (for more recent different model approaches concerning the multifluid properties of the solar wind termination shock see Zank et al. 1993; Chalov \& Fahr 1994, 1995, 1996; Zank 1999; Fahr \& Scherer 2005). For a magnetized plasma, the jump conditions are usually used in the form given by Serrin (1959), Zel'dovich \& Raizer (1966), Landau \& Lifshitz (1977) or Diver (2001). However, there are several problems with the MHD treatment of the shock. First and foremost, this approach only works for collision-dominated shocks, guaranteeing an effective equilibrium of the distribution functions. But there are many kind of shocks where this condition is not fulfilled, such as solar wind bowshocks found near astrophysical objects (comets or planets), shocks forming in corotating interaction regions, traveling interplanetary shocks, or the heliospheric termination shock.

Another, less popular problem related to the MHD treatment of astrophysical shocks is the fact that the jump conditions for the lower moments of the distribution function are underdetermined, which means that there remains one variable that cannot be determined by the set of jump conditions alone. Usually, this variable is selected to be the downstream pressure anisotropy, $\lambda=p_{\perp} / p_{\|}$, since in most physical situations, the dependence of the system on this parameter is very weak (see, e.g. Erkaev et al. 2000). Nevertheless, the MHD description of a shock transition is not complete; in particular, the dissipation mechanisms producing entropy are not specified.
Finally, MHD is unable to provide the full velocity distribution function of the downstream particles, since only the knowledge of all velocity moments is equivalent to the knowledge of the distribution function itself, while MHD is characterized by only a few, low-order downstream moments. In addition, any physical plasma flow must be electrically neutral on average, which requires (at least) the presence of two different types of particles, ions, and electrons. Attempts to solve this problem numerically by applying shock simulation calculations within hybrid or full particle codes (e.g., see Hada et al. 2003; Scholer et al. 2003), despite being computationally highly pretentious, most often do not offer a stationary solution for the shock profile, but due to accumulation of specularly reflected ions upstream of the shock cause the formation and repeated reformation of ramp shocks on timescales of gyroperiods of the ramp magnetic fields. While for unrealistic ion/electron mass ratios (i.e., $m_{\mathrm{i}} / m_{\mathrm{e}}=60$ ) the average shock profile attains a relatively regular shape, for realistic ratios $m_{\mathrm{i}} / m_{\mathrm{e}}=1840$ a completely erratic profile is found. Most unclear in all the simulation runs remains the role of shockheated electrons. Hybrid simulation codes show that reasonable results can only be achieved by them for unreasonably high electron resistivities (Scudder 1995), which perhaps could be ascribed to ion-acoustic instabilities or to Coulomb collision mediation, but as could be shown in particle-particle simulations, neither ion-acoustic instabilities nor Coulomb collision effects are likely to become of the required importance (see Wilkinson 1991; Thomsen et al. 1985).

In face of these difficulties, we have recently developed a completely different approach, based on kinetic equations (Fahr $\&$ Siewert 2006). We have derived the kinetic Boltzmann equation for a collisionless plasma and the corresponding velocity distribution function, and were able to demonstrate that at least for a purely perpendicular shock (where the shock normal is orthogonal to the magnetic field) this approach does not conflict 
with the predictions from common high Mach-number MHD. In addition, we have demonstrated that this approach provides the missing relation required to completely solve the anisotropic jump conditions. In this publication we intend to build upon our earlier results, deriving the complete downstream distribution function, and, correspondingly, a full set of relations between all upstream and downstream moments. Knowing the downstream distribution function would be useful, since there may be other velocity moments of relevance for physics than the pressures and densities. Furthermore, instabilities and electromagnetic radiation processes from the shock are sensitive to the specific distribution function.

\section{The downstream distribution function}

\subsection{The Boltzmann equation}

In a recent paper (Fahr \& Siewert 2006) we derived the Boltzmann equation for the distribution function $f\left(w_{\|}, w_{\perp}, s\right)$ of a collisionless magnetized plasma crossing an MHD shock, starting from the most general, relativistic form of the Boltzmann equation, where $w_{\|, \perp}$ is the velocity of the individual plasma particles, with the subscript denoting the orientation of the velocity components with respect to the magnetic field. The equation is taken in the accelerated, co-moving reference frame of the plasma bulk, moving with the bulk velocity $U(s)$, and $s$ is the streamline coordinate in the direction of the shock normal. In addition, in this derivation the only assumption on the transition region was that the gyration of particles may be averaged out, i.e., that the bulk velocity $U_{n}$ and the magnetic field $\boldsymbol{B}$ change sufficiently slowly. In the most general situation, as we have shown, the Boltzmann equation takes the form

$\frac{\mathrm{d}}{\mathrm{d} s} f\left(w_{\|}, w_{\perp}, s\right)=A_{\|} \frac{\mathrm{d}}{\mathrm{d} w_{\|}} f\left(w_{\|}, w_{\perp}, s\right)+A_{\perp} \frac{\mathrm{d}}{\mathrm{d} w_{\perp}} f\left(w_{\|}, w_{\perp}, s\right)$

with

$$
\begin{aligned}
& A_{\|}=-\frac{U_{n}}{U_{n}+w_{\|} \cos \alpha}\left(\cos \alpha \frac{\mathrm{d} U_{n}}{\mathrm{~d} s}+w \frac{\mathrm{d}}{\mathrm{d} s} \cos \alpha\right) \\
& A_{\perp}=-\frac{U_{n}}{U_{n}+w_{\|} \cos \alpha}\left(\frac{w_{\perp}}{2 B} \frac{\mathrm{d} B}{\mathrm{~d} s}+w \frac{\mathrm{d}}{\mathrm{d} s} \sin \alpha\right),
\end{aligned}
$$

where $\alpha$ is the angle between the magnetic field $B$ and the shock normal and $U_{n}$ is the normal component of the bulk velocity of the plasma. We note that these quantities are usually not constant, but functions of $s$ in the transition region of the shock, and we would also like to emphasize that this equation is taken in the accelerated, co-moving frame of the plasma, where explicit acceleration terms need to be taken into account, but without applying any details of the deceleration mechanism, which is a highly non-trivial situation.

In Fahr \& Siewert (2006), we have also demonstrated that many MHD properties of the plasma do not depend on the specific fine structure of the shock transition region itself, even when treated using our Boltzmann approach. This includes the relations

$$
\begin{aligned}
\rho_{2} & =x \rho_{1} \\
p_{\| 2} & =x p_{\| 1} \\
p_{\perp 2} & =x^{2} p_{\perp 1},
\end{aligned}
$$

where the indices 1 and 2 correspond to the up- and downstream quantities and $x$ is the MHD compression ratio, which is valid for all physical upstream distribution functions. Equation (4) represents the well-known conservation of the MHD mass flux, which automatically follows from the MHD treatment of the shock, while the other two equations are a new result, which has not been derived before, since the MHD jump conditions are underdetermined.

These relations for the partial pressures may be understood in terms of the two adiabatic invariants related to the underlying anisotropic pressure model (Chew et al. 1956),

$$
\begin{aligned}
\frac{\mathrm{d}}{\mathrm{d} t}\left(\frac{p_{\|} B^{2}}{\rho^{3}}\right) & =0 \\
\frac{\mathrm{d}}{\mathrm{d} t}\left(\frac{p_{\perp}}{B \rho}\right) & =0
\end{aligned}
$$

from which it is possible to derive two additional jump conditions, namely

$$
\begin{array}{r}
{\left[\left[\frac{p_{\|} B^{2}}{\rho^{3}}\right]\right]=0} \\
{\left[\left[\frac{p_{\perp}}{B \rho}\right]\right]=0,}
\end{array}
$$

although these conditions are usually ignored in literature, which subsequently leads to an underdermined set of jump conditions. While these relations have already been derived by Chew et al. (1956), we still feel inclined to sketch an alternative way to arrive there, especially considering that we have not yet made any specific assumptions about $U(s)$ and $\boldsymbol{B}(s)$. Based on the fact that the terms in the Boltzmann equation are of the form $a(s) \mathrm{d} b(s) / \mathrm{d} s$, we have derived the identity (Fahr \& Siewert 2006)

$\int_{s_{1}}^{s_{2}} a(s) \frac{\mathrm{d} b(s)}{\mathrm{d} s} \mathrm{~d} s=\frac{1}{2}\left(a_{2}+a_{1}\right)\left(b_{2}-b_{1}\right)$,

for arbitrary (integrable) functions $f(s)$ and $g(s)$ using a variant of the well known symmetrical-antisymmetrical decomposition of the functions,

$$
\begin{aligned}
{[f, g](s)=} & {[f, g]_{1}+\left([f, g]_{2}-[f, g]_{1}\right) } \\
& \times\left(\frac{1}{2}+[f, g]_{s}(s)+[f, g]_{a}(s)\right) .
\end{aligned}
$$

This indicates that the distribution function $f_{2}(w)$ does not depend on the fine structure of the transition region itself. We would also like to point out that this relation does not hold in the special case where one of the functions is perfectly symmetrical or antisymmetrical with respect to the mentioned decomposition, i.e., $[f, g]_{[s, a]} \equiv 0$. Considering that in a physical situation, there is no perfect (anti-)symmetry, we may identify $\frac{\mathrm{d}}{\mathrm{d} t}=U(s) \frac{\mathrm{d}}{\mathrm{d} s}$, and one automatically obtains the mentioned additional jump conditions. This result also allows us to derive the downstream MHD velocity moments, by multiplying Eq. (1) with any power of $w_{\|, \perp}$ and integrating over $\mathrm{d}^{3} w \mathrm{~d} s$, which is equivalent to the knowledge of the distribution function.

We want to point out that, for a non-perpendicular shock, the differential bulk velocity of the plasma may be identified as the factor $U_{n}+w_{\|} \cos \alpha$ in the Boltzmann Eq. (1). Under this configuration the individual particles cross the shock with differential bulk velocities, no longer depending on the streamline coordinate $s$ only, but also on their parallel velocity $w_{\|}$. This effect considerably increases the mathematical and physical difficulties related to a full solution of the Boltzmann equation, by introducing a singularity at $U_{n}+w_{\|} \cos \alpha=0$. This may be 
physically interpreted as some particles crossing the shock into the "wrong" direction (i.e., from the downstream side to the upstream one), which might happen on account of some particles being reflected from the potential barrier responsible for the deceleration of the plasma. We were able to prove (Fahr \& Siewert 2006) that in this situation and for practically all physical distribution functions $f(w, s)$ (i.e., sufficiently smooth functions), a straightforward integration of the Boltzmann equation across the shock under these conditions does not lead to physical results.

\subsection{The purely perpendicular shock}

Now we solve the Boltzmann equation for the full downstream plasma distribution function. In the case of the purely perpendicular shock, Eq. (1) simplifies to

$\frac{\mathrm{d} f}{\mathrm{~d} s}=-\frac{w_{\perp}}{2 B(s)} \frac{\mathrm{d} B(s)}{\mathrm{d} s} \frac{\mathrm{d} f}{\mathrm{~d} w_{\perp}}=w_{\perp} \alpha(s) \frac{\mathrm{d} f}{\mathrm{~d} w_{\perp}}$,

with

$$
\begin{aligned}
\alpha(s) & =-\frac{1}{2 B(s)} \frac{\mathrm{d} B(s)}{\mathrm{d} s} \\
& =-\frac{1}{2} \frac{\mathrm{d}}{\mathrm{d} s} \ln B(s) .
\end{aligned}
$$

Since Eq. (13) is a linear partial differential equation, we try to solve this expression with a separation Ansatz,

$f\left(w_{\|}, w_{\perp}, s\right)=f_{\|}\left(w_{\|}\right) f_{\perp}\left(w_{\perp}\right) f_{s}(s)$.

Since Eq. (13) does not depend on $w_{\|}$, we automatically get

$f_{\|, 1}=f_{\|, 2}$,

where the indices 1 and 2 denote the up- and downstream distribution functions. We would like to point out that this expression seems, at the first glance, to conflict with Eq. (5), but, as we will demonstrate in Sect. 2.4, this is not the case, as the MHD compression factor is a result of a general phase space transformation. We would also like to emphasize that the separation Ansatz usually fails for arbitrary angles $\alpha$, since in this situation the variables $s$ and $w_{\|, \perp}$ may not be separated, and other popular representations likewise seem to fail. For an overview of methods to solve several specific model cases of the Boltzmann equation, see, e.g., Cercignani (1988).

Inserting Eq. (15) into Eq. (13), the Boltzmann equation separates into two ordinary differential equations

$$
\begin{aligned}
\frac{\mathrm{d}}{\mathrm{d} s} f_{s} & =\lambda \alpha(s) f_{s} \\
\frac{\mathrm{d}}{\mathrm{d} w_{\perp}} f_{\perp} & =\frac{\lambda}{w_{\perp}} f_{\perp},
\end{aligned}
$$

where $\lambda$ is the separation parameter, which is an arbitrary number. These equations can be solved without difficulties and we obtain

$$
\begin{aligned}
f_{s} & =\exp \left(\int_{s_{1}}^{s} \lambda \alpha(\hat{s}) \mathrm{d} \hat{s}\right) \\
f_{\perp} & =\exp \left(\int_{w_{\perp, 0}}^{w_{\perp}} \frac{\lambda}{\hat{w}_{\perp}} \mathrm{d} \hat{w}_{\perp}\right) \\
& =\exp \left(\lambda \ln \left(w_{\perp}\right)\right) \\
& =w_{\perp}^{\lambda} .
\end{aligned}
$$

Since the Boltzmann equation is linear, its most general solution is a linear combination of the individual "building blocks",

$$
\begin{aligned}
f\left(w_{\|}, w_{\perp}, s\right) & =f_{\|} \cdot \sum_{n} a_{n} w_{\perp}^{\lambda_{n}} \exp \left(\int_{s_{1}}^{s} \lambda_{n} \alpha(\hat{s}) \mathrm{d} \hat{s}\right) \\
& =f_{\|} \cdot \int \mathrm{d} \lambda a(\lambda) w_{\perp}^{\lambda} \exp \left(\int_{s_{1}}^{s} \lambda \alpha(\hat{s}) \mathrm{d} \hat{s}\right),
\end{aligned}
$$

with the arbitrary coefficients $a_{n}$ and $\lambda_{n}$ and the arbitrary function $a(\lambda)$.

We now need to specialize this solution to our problem, which requires fine-tuning the $a_{n}$ and $\lambda_{n}$ in such a way that the upstream distribution function is reproduced in the upstream region. In this case we get

$f_{s, 1}=1$,

which leads to

$f\left(w_{\|}, w_{\perp}\right)_{1}=f_{\|} \cdot \sum_{i} a_{n} w_{\perp}^{\lambda_{n}}$.

This is the power-law expansion of the partial upstream distribution function $f_{\perp}$, from which we automatically obtain

$\lambda_{n}=n$

$a_{n}=\frac{1}{n !} \frac{\mathrm{d}^{n}}{\mathrm{~d} w_{\perp}^{n}} f_{\perp}\left(w_{\perp}=0\right)$.

For this reason, we will see the upstream distribution function, for the rest of this analysis, as a discrete sum (Eq. (21)),

$f\left(w_{\|}, w_{\perp}, s\right)=f_{\|} \cdot \sum_{n} a_{n} w_{\perp}^{n} \exp \left(n \int_{s_{1}}^{s} \alpha(\hat{s}) \mathrm{d} \hat{s}\right)$.

Before we consider the integral of the streamline coordinate $s$ over the shock, we investigate the range of validity for the powerlaw Ansatz. The convergence of an infinite sum, such as a powerlaw expansion, requires that the coefficients $a_{n}$ converge to zero "fast enough". Analysis provides several conditions that may be applied to verify this property. In addition, from basic physical concepts, we know that any distribution function must be normalizable, which is typically ensured by a Maxwellian representation,

$f(w)=g(w) \cdot \exp \left(-\frac{w^{2}}{\Theta^{2}}\right)$.

The Taylor expansion of the exponential function $\exp (w)$ is

$\exp (w)=\sum_{k=0}^{\infty} \frac{1}{k !} w^{k}$

which is absolutely convergent on the entire real axis (see, e.g., Kaballo 1996, Chap. 11 and V). From this it automatically follows that the substitution

$w \rightarrow-w^{2}$

also results in a convergent expansion. Then it follows from Mertens's theorem on the product of infinite sums (Kaballo 1996, Chap. 32) that, as long as the Taylor expansion of $g(w)$ converges, the product of both sums also converges. So, for a simple Maxwellian distribution function, multiplied by any realanalytical function, the power-law expansion Ansatz is viable and valid. 
Now, all that remains to do is to evaluate the integral in the exponent, which seems to depend on the fine structure of the shock, as a function of the streamline coordinate $s$. However, this expression can be evaluated by assuming that, usually, one is not interested in the fine structure of the shock itself, but only in the asymptotic distribution functions on the far upstream $\left(s=s_{1}\right)$ and downstream $\left(s=s_{2}\right)$ sides. In this situation it follows that

$$
\begin{aligned}
\int_{s_{1}}^{s_{2}} \mathrm{~d} s \alpha(s) & =-\frac{1}{2} \int_{s_{1}}^{s_{2}} \mathrm{~d} s \frac{1}{B(s)} \frac{\mathrm{d}}{\mathrm{d} s} B(s) \\
& =-\frac{1}{2} \int_{s_{1}}^{s_{2}} \mathrm{~d} s \frac{\mathrm{d}}{\mathrm{d} s} \ln (B(s)) \\
& =-\frac{1}{2}\left(\ln B_{2}-\ln B_{1}\right) \\
& =-\frac{1}{2} \ln \frac{B_{2}}{B_{1}} \\
& =-\frac{1}{2} \ln x .
\end{aligned}
$$

Inserting this result in Eq. (26) we obtain

$$
\begin{aligned}
f_{2}\left(w_{\|}, w_{\perp}\right) & =f_{\|} \cdot \sum_{n} a_{n} w_{\perp}^{n} \exp \left(-\frac{n}{2} \ln (x)\right) \\
& =f_{\|} \cdot \sum_{n} a_{n} w_{\perp}^{n} x^{-n / 2} \\
& =f_{\|} \cdot \sum_{n} a_{n}\left(\frac{w_{\perp}}{\sqrt{x}}\right)^{n} .
\end{aligned}
$$

From this we immediately see that the downstream distribution function is, except for a modification of the variable $w_{\|}$, the same as the upstream distribution function, namely

$$
f_{2}\left(w_{\|}, w_{\perp}\right)=f_{1}\left(w_{\|}, \bar{w}_{\perp}=\frac{w_{\perp}}{\sqrt{x}}\right) .
$$

This is an analytical relation, which does not require any additional, complicated numerical treatment of the Boltzmann equation. A few examples for upstream and downstream distribution functions are presented in Figs. 1 and 2. While it has been known for a long time that the Boltzmann equation preserves the functional form of the initial function, we have nevertheless proven that, for a purely perpendicular shock, it is possible to write down this explicit solution without any further numerical considerations.

\subsection{Similar results in recent literature}

\subsubsection{Isotropic distributions}

Equation (32) is very similar to the result by Fahr \& Lay (2000, Eq. (13)),

$f_{2, \mathrm{FL}}\left(w^{2}\right)=\frac{1}{\sqrt{x}} f_{1, \mathrm{FL}}\left(\frac{w^{2}}{x}\right)$,

which has been derived for an isotropic plasma using the conservation of the magnetic moment and the phase space volume. The additional factor of $x^{-1 / 2}$ is a result of differences in the phase spaces for an isotropic and an anisotropic plasma. The authors of the mentioned publication derived the relation

$$
f_{2, \mathrm{FL}}\left(w^{2}\right)=x J f_{1, \mathrm{FL}}\left(\frac{w^{2}}{x}\right),
$$

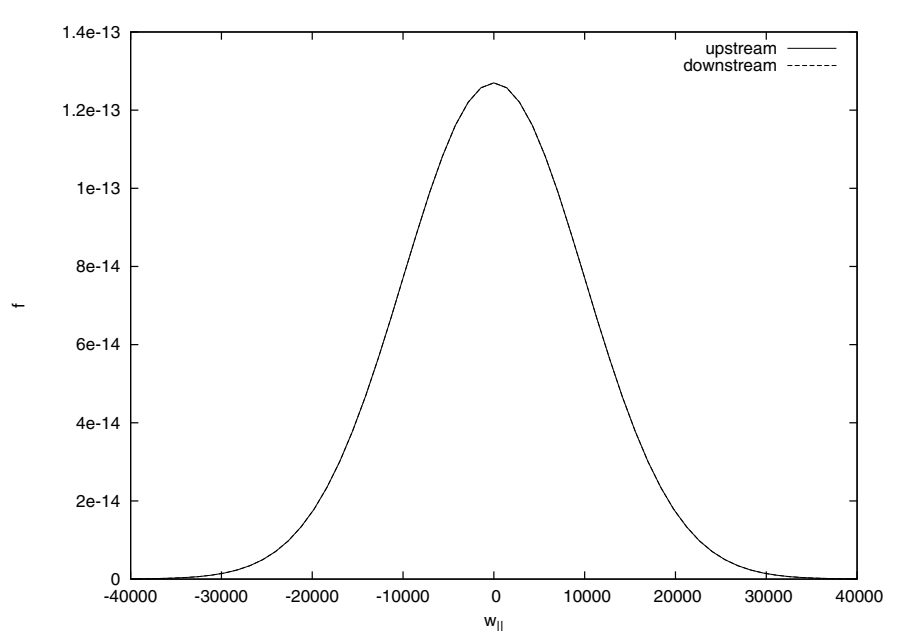

Fig. 1. Upstream and downstream distribution functions for an isotropic upstream Maxwell distribution function. Cut in the $w_{\perp}=0$-plane. Both curves are identical, demonstrating that the functional dependence on $w_{\|}$does not change across the shock.

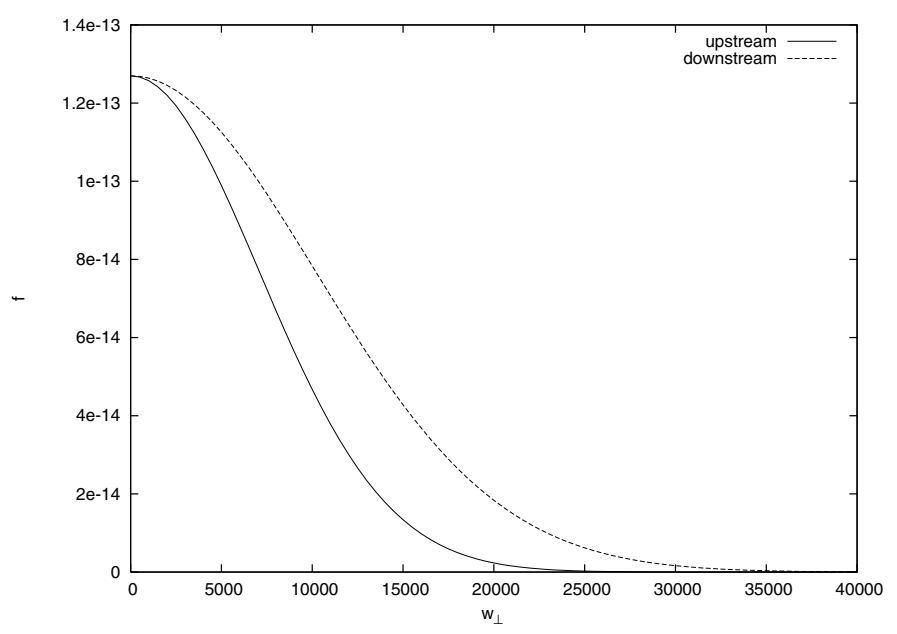

Fig. 2. Upstream and downstream distribution functions for an isotropic upstream Maxwell distribution function. Cut in the $w_{\|}=0$-plane, the MHD compression ratio is $x \simeq 2.05$.

where $J$ is the Jacobian of the phase space transformation, which is

$J_{\text {iso }} \propto \frac{v_{1}^{2}}{v_{2}^{2}} \frac{\mathrm{d} v_{1}}{\mathrm{~d} v_{2}}=x^{-3 / 2}$

for an isotropic plasma, but

$J_{\text {aniso }} \propto \frac{v_{\perp 1}}{v_{\perp 2}} \frac{\mathrm{d} v_{\perp 1}}{\mathrm{~d} v_{\perp 2}}=x^{-1}$

for an anisotropic one. From this relation we learn that the basic argument applied by Fahr \& Lay (2000) is in good agreement with the Boltzmann-kinetic treatment presented here.

However, there is one fundamental difference between these two calculations. In an earlier publication (Fahr \& Siewert 2006), we demonstrated that

$\lambda_{2}=x \lambda_{1}$

which means that as long as a shock does exist (i.e., $x>1$ ), any isotropic upstream distribution must automatically become 
anisotropic downstream. Instead, Fahr \& Lay (2000) have assumed that, due to effective pitch angle scattering,

$\lambda_{2, \mathrm{FL}}=\lambda_{1, \mathrm{FL}}=1$,

which means that any isotropic upstream distribution will still be isotropic on the downstream side. As we demonstrate in the next section, Eq. (37) still holds, so that, without the additional inclusion of strong relaxation processes, assumption 38 must be considered wrong. Finally, we note that although the downstream plasma may in principle be re-isotropized after crossing the shock, no matter what the present kinetic treatment of the shock transition itself predicts, it is still rather unlikely that the relation in Eq. (33) holds, as the re-isotropization is completely unrelated to the processes in the transition region itself, especially if the additional processes break the conservation of the magnetic moment.

\subsubsection{Power-law distributions}

The result from Eq. (32) in some respects seems to support the recent derivation given by Fisk \& Gloeckler (2006), who found a general power law,

$f \propto w^{n}$,

with a power index of $n=-5$, governing all distribution functions of suprathermal ions that can resonate with compressional magnetoaccoustic turbulences in the quiet periods of the solar wind. Taking this initial upstream spectrum and sending it through a perpendicular shock, one obtains

$f_{\perp} \propto\left(\frac{w_{\perp}}{\sqrt{x}}\right)^{n}=x^{-n / 2} w_{\perp}^{n}$,

which means that a power-law spectrum is conserved even across a shock, including the numerical value of the exponent. It should, however, be recognized that the mentioned authors assume isotropic distribution functions, an isotropic $k$-vector distribution of the turbulence, and that a stationary equilibrium situation can be established, where the same amount of energy is resonantly absorbed by ions from the turbulences as work of the ion pressure against the compressional fluctuations is done. At first view, our concept is not at all based on these special conditions to be fulfilled. In addition, since many astrophysical particle spectra are well parameterized by power laws, including (but not limited to) the basic (Fermi) shock acceleration mechanism (see, e.g. Pohl 2002), we may already conclude that our result does not contradict observations.

\subsection{Downstream particle moments}

To further verify our result, we now validate the three momentum relations (Eqs. (4)-(6))

$$
\begin{aligned}
n_{2} & =x n_{1} \\
p_{\|, 2} & =x p_{\|, 1} \\
p_{\perp, 2} & =x^{2} p_{\perp, 1},
\end{aligned}
$$

which have been verified independently of the form of $f_{1}$ and $f_{2}$ Fahr \& Siewert (2006). First, we note that

$$
\begin{aligned}
\int \mathrm{d}^{3} w f_{1}\left(w_{\|}, \frac{w_{\perp}}{\sqrt{x}}\right) & =2 \pi \int \mathrm{d} w_{\|} w_{\perp} \mathrm{d} w_{\perp} f_{1}\left(w_{\|}, \bar{w}_{\perp}\right) \\
& =2 \pi x \int \mathrm{d} w_{\|} \bar{w}_{\perp} \mathrm{d} \bar{w}_{\perp} f_{1}\left(w_{\|}, \bar{w}_{\perp}\right) .
\end{aligned}
$$

From this expression it automatically follows that Eq. (4) is valid.

For the partial pressures we first recall that

$$
\begin{aligned}
& p_{\|}=\left\langle m w_{\|}^{2}\right\rangle \\
& p_{\perp}=\left\langle m w_{\perp}^{2}\right\rangle .
\end{aligned}
$$

Since $f_{\|, 2}$ is not modified by the shock (Eq. (16)), it automatically follows that

$p_{\|, 2}=x p_{\|, 1}$.

For the perpendicular pressure we get the integral

$$
\begin{aligned}
p_{\perp, 2} & =\int \mathrm{d}^{3} w w_{\perp}^{2} f_{1}\left(w_{\|}, \bar{w}_{\perp}\right) \\
& =m x \int \mathrm{d}^{3} w \bar{w}_{\perp}^{2} f_{1}\left(w_{\|}, \bar{w}_{\perp}\right) \\
& =m x^{2} \int \mathrm{d}^{3} \bar{w} \bar{w}_{\perp}^{2} f_{1}\left(w_{\|}, \bar{w}_{\perp}\right) \\
& =x^{2} p_{\perp, 1}
\end{aligned}
$$

These are exactly the expected results, which demonstrates that the explicit form of the downstream function (Eq. (32)) is correct.

We note that it is possible to generalize the above results to arbitrary velocity moments, by

$$
\begin{aligned}
a_{i j, 2} & =\left\langle w_{\|}^{i} w_{\perp}^{j}\right\rangle \\
& =\int \mathrm{d}^{3} w w_{\|}^{i} w_{\perp}^{j} f_{1}\left(w_{\|}, \bar{w}_{\perp}\right) \\
& =x \int \mathrm{d}^{3} \bar{w} w_{\|}^{i} w_{\perp}^{j} f_{1}\left(w_{\|}, \bar{w}_{\perp}\right) \\
& =x \cdot x^{j / 2} \int \mathrm{d}^{3} \bar{w} w_{\|}^{i} \bar{w}_{\perp}^{j} f_{1}\left(w_{\|}, \bar{w}_{\perp}\right) \\
& =x^{1+j / 2} a_{i j, 1},
\end{aligned}
$$

which, again, does not depend on the form of the distribution function or the fine structure of the transition region. This result should be interesting for many MHD calculations, since the jump conditions alone generally do not allow for a full determination of all relevant downstream MHD moments. In addition, this result proves that the phase space argument from Fahr \& Lay (2000) is valid even under anisotropic conditions. This result is also in good agreement with the well-known fact that the knowledge of the distribution function is equivalent to the knowledge of all velocity moments.

If we repeat this procedure for Eq. (33) (where it was assumed that both the upstream and downstream sides are completely isotropic), we obtain

$a_{i j, 2, \mathrm{FL}}=x^{1+i / 2+j / 2} a_{i j, 1}$,

which directly follows from the relations

$$
\begin{array}{ll}
w_{\|}=w \cos \theta & w_{\perp}=w \sin \theta \\
\bar{w}_{\|}=\sqrt{x} w \cos \theta & \bar{w}_{\perp}=\sqrt{x} w \sin \theta .
\end{array}
$$

It should be noted that this expression is consistent both with the MHD compression relation and with the assumption that the system is isotropic both on the upstream and downstream sides. This again proves that the phase space argument applied by (Fahr \& Lay 2000) is, in principle, correct, and that the anisotropy has to be assumed explicitly. 


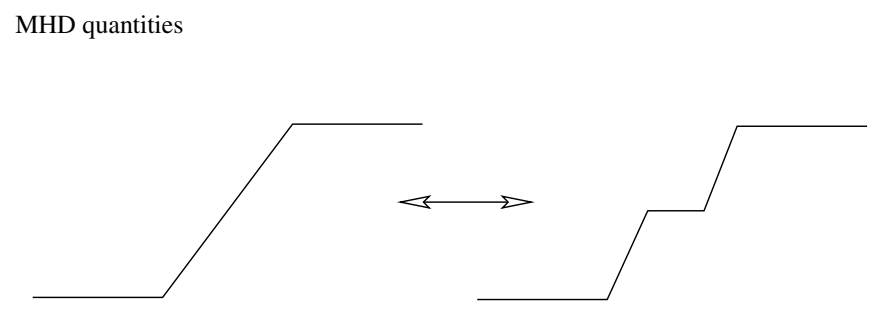

Fig. 3. If we cut the shock in two parts, and move these parts away from each other, there are still MHD quantities present inside the shock. A single shock is mathematically equivalent to two (or more) distinct shocks, and the form of the distribution function will remain the same inside the transition region.

\section{More details about the perpendicular shock}

\subsection{The entropy}

From the third hauptsatz of thermodynamics it is known that the entropy in a given system will always increase towards a maximum. The total entropy inside an anisotropic plasma crossing a shock is given by Erkaev et al. (2000)

$S=\frac{k}{2} \ln \left(\frac{p_{\perp}^{2} p_{\|}}{\rho^{5}}\right)$.

This expression is based on the two adiabatic laws governing a collisionless magnetized plasma (Eqs. (7) and (8)).

We now apply Eq. (46) and obtain

$S_{2}=\frac{k}{2} \ln \left(\frac{\left(x^{4} p_{\perp}^{2}\right)\left(x p_{\|}\right)}{(x \rho)^{5}}\right)$

for the downstream entropy for a perpendicular shock, and

$S_{2}-S_{1}=0$

for the total entropy change during the passage through the shock, which means that as long as the kinetic energy in the system is conserved, the entropy is conserved as well. This relation considerably simplifies the identification of dissipation processes, which are physically significant in causing the entropy to grow. This result contradicts the results obtained by Erkaev et al. (2000), who claim that the variation of the entropy throughout the shock must be positive on account of an energy flux from the parallel component to the perpendicular one (or vice versa). While this point is, in principle, correct, our zero-result demonstrates that there is no such energy flow between parallel and perpendicular components for a simple nondissipative shock.

\subsection{The fine structure of the shock}

Most radiation processes are very sensitive to the fine structure of the plasma distribution function. Since we have demonstrated that, at least for a purely perpendicular shock, the downstream plasma can be derived almost trivially from the upstream plasma, we now investigate what may happen to the distribution function inside the transition region. We start with a Gedanken experiment. We divide the initial shock into two separate areas (see Fig. 3), where the transition is no longer

$f_{1} \rightarrow f_{2}$

but

$f_{1} \rightarrow f_{1 a} \rightarrow f_{2}$.
Obviously, we may take both subshocks and pull them apart an arbitrary distance, which is mathematically equivalent to a single shock as the Boltzmann equation equals zero between these two halves. On the other hand, our formalism may be applied to each of these two subshocks separately. From this it follows that, at an arbitrary point inside the transition region, the distribution function will have the same form as in the far up- and downstream regions, and the distribution function will follow Eq. (32) even inside the transition region, by using varying MHD parameters $x$ and $U_{n 1}$. This also means that any wave-generating mechanism must already be present on the upstream side of the shock, since the shock does not modify the specific form of the velocity distribution, nor does it introduce any exclusive, "new" mechanisms, at least as long as MHD approximately holds inside the transition region.

\subsection{The form of the plasma flow}

In general, most, if not all astrophysical plasma flows are not exactly one-dimensional, but they expand during their motion through space; the solar wind is, at least in first approximation, radially symmetric with respect to the sun itself. This effect usually results in a small nonstationarity, like the one mentioned in the preceding section. However, if the opening angle is sufficiently small, then the correction to a perfectly parallel shock can be safely ignored. We now give a quantitative estimate for this. A surface element on a sphere is given by

$\mathrm{d} A=R^{2} \mathrm{~d} \Omega$.

If we consider two surface elements at a relative distance $d$, such as the borders of a shock transition region, then the deviation from the perfectly parallel plasma flow can be estimated by

$$
\begin{aligned}
\mathrm{d} A_{2}-\mathrm{d} A_{1} & =\left(\left(R+\frac{d}{2}\right)^{2}-\left(R-\frac{d}{2}\right)^{2}\right) \mathrm{d} \Omega \\
& =2 R d \mathrm{~d} \Omega .
\end{aligned}
$$

To estimate the smallness of this expression, we determine the relative "fluctuation" in the surface element by dividing Eq. (57) by 55 , which leads to the condition

$\frac{d}{R} \ll 1$

So, for a (quasi-)radially expanding system, as long as we are far away from the origin and the transition region of the shock is sufficiently small, then the plasma flow may be considered to be parallel, and the interaction between the charged particles and the MHD waves is, effectively, restricted to the shock. This condition should be fulfilled for most astrophysical shocks in the solar system.

\section{Conclusions}

In this paper we have solved the Boltzmann equation of a collisionless plasma crossing a perpendicular MHD shock. It is possible to give a simple analytical solution to the downstream distribution function (Eq. (32)), as long as the upstream distribution and the MHD compression ratio are known, without the need for complicated numerical simulations, which are getting increasingly popular in these days (see, e.g., Hada et al. 2003; Scholer et al. 2003). Similarly, it is possible to derive all of the downstream moments as analytical functions of the upstream ones and the MHD compression ratio $x$, which provides an excellent test 
case for numerical simulations of shocks in plasmas. In the future, we hope to be able to give similar results for the parallel and inclined shocks, using a different mathematical Ansatz. In addition, we are currently preparing an in-depth investigation of the influence of additional terms on the Boltzmann equation, such as the inclusion of wave-particle interaction terms, turbulence terms, or Fokker-Planck type diffusion.

Acknowledgements. We are grateful to the DFG for financial support within the frame of the DFG-Project Fa 97/31-1.

\section{References}

Cercignani, C. 1988, The Boltzmann Equation and Its Applications (New York: Springer-Verlag)

Chalov, S. V., \& Fahr, H.-J. 1994, A\&A, 288, 973

Chalov, S. V., \& Fahr, H.-J. 1995, Planet. Space Sci., 43, 1035

Chalov, S. V., \& Fahr, H.-J. 1996, A\&A, 311, 317

Chew, G. F., Goldberger, M. L., \& Low, F. E. 1956, Proc. R. Soc. London A, 236, 112

Diver, D. A. 2001, A Plasma Formulary for Physics, Technology and Astrophysics (Hoboken, New Jersey: John Wiley)
Erkaev, N. V., Vogl, D. F., \& Biernat, H. K. 2000, J. Plasma Physics, 64, 561 Fahr, H.-J., \& Lay, G. 2000, A\&A, 356, 327

Fahr, H.-J., \& Scherer, K. 2005, J. Geophys. Res., 110, A02103

Fahr, H.-J., \& Siewert, M. 2006, A\&A, 458, 13

Fisk, L. A., \& Gloeckler, G. 2006, ApJ, 640, L79

Hada, T., Onishi, M., Lembege, B., \& Savoinin, P. 2003, J. Geophys. Res., 108, 1233

Kaballo, W. 1996, Einführung in die Analysis I (Heidelberg, Berlin, Oxford: Spektrum Akademischer Verlag)

Landau, L. D., \& Lifshitz, E. M. 1977, Lehrbuch der theoretischen Physik (Berlin: Akademiker-Verlag)

Pohl, M. 2002, Einführung in die Hochenergieastrophysik (Aachen: Shaker Verlag)

Scholer, M., Shinohara, I., \& Matsukiyo, S. 2003, J. Geophys. Res., 108, 1014

Scudder, J. D. 1995, Adv. Space Res., 15, 181

Serrin, J. 1959, Handbuch der Physik, Vol. VIII, Mathematical principles of classical fluid mechanics (Berlin: Springer Verlag), 125

Thomsen, M. F., Gosling, J. T., Bame, S. J., \& Mellot, M. M. 1985, J. Geophys. Res., 90, 137

Wilkinson, W. P. 1991, J. Geophys. Res., 96, 17675

Zank, G. P. 1999, Space Sci. Rev., 89, 413

Zank, G. P., Webb, G. M., \& Donohue, D. J. 1993, ApJ, 406, 97

Zel'dovich, Y. B., \& Raizer, Y. P. 1966, Physics of shock waves and high-temperature hydrodynamic phenomena (New York: Academic Press), 75 